\title{
Prospects of non-resonant and resonant Higgs pair production at the HL-LHC
}

\section{Amit Adhikary, ${ }^{a, *}$ Shankha Banerjee, ${ }^{b}$ Rahool Kumar Barman, ${ }^{c}$ Biplob Bhattacherjee $^{d}$ and Saurabh Niyogi ${ }^{e}$}

${ }^{a}$ Institute of Theoretical Physics, Faculty of Physics, University of Warsaw, Pasteura 5, PL 02-093, Warsaw, Poland

${ }^{b}$ CERN, Theoretical Physics Department, CH-1211 Geneva 23, Switzerland

${ }^{c}$ Department of Physics, Oklahoma State University, Stillwater, Oklahoma, 74078, USA

${ }^{d}$ Centre for High Energy Physics, Indian Institute of Science, Bangalore 560012, India

e Gokhale Memorial Girls' College, 1/1, Harish Mukherjee Road, Kolkata 700020, India

E-mail: amit.adhikary@fuw.edu.pl

We combined multiple final states ensuing from non-resonant Higgs pair production by optimising over each final state using multivariate techniques and put bounds on the self-coupling of Higgs by employing the log-likelihood confidence level hypothesis test. Further, we calculate the production cross-section limits from multifarious heavy Higgs decay channels, including resonant di-Higgs production, in a model-independent way and draw its impact on the MSSM parameter space at the HL-LHC.

*** The European Physical Society Conference on High Energy Physics (EPS-HEP2021), ***

*** 26-30 July $2021 * * *$

*** Online conference, jointly organized by Universität Hamburg and the research center DESY ***

${ }^{*}$ Speaker 


\section{Introduction}

The measurement of Higgs boson self-coupling is among the next goal of the Large Hadron Collider (LHC) era. It can verify the nature of Higgs potential and thereby the electroweak symmetry breaking (EWSB) mechanism. However, a direct measurement of this coupling requires an observation of Higgs pair or di-Higgs production, $p p \rightarrow h h$. This observation becomes challenging because of the small di-Higgs production rate in Standard Model (SM). At the LHC, the dominant production happens via the gluon fusion process with a triangle and box diagram, and there is destructive interference between these two diagrams. Our motive is to analyse various Higgs pair production channels at the HL-LHC and infer the potential of HL-LHC in di-Higgs observation [1].

We are now aware of beyond the SM (BSM) physics which is a requirement to understand various unexplained phenomena in SM. One such well-motivated BSM model to explore new particle searches is the Minimal Supersymmetric Standard Model (MSSM). We investigate the Higgs sector of MSSM in our work [2]. Here, we specifically search for MSSM Higgs bosons in various final states and evaluate the reach of HL-LHC in the MSSM parameter space.

\section{The non-resonant Higgs pair production}

Here, the term "non-resonant" refers to the Higgs pair production in SM. Multifarious Higgs decay modes constitute phenomenologically rich di-Higgs final states. We select the final states which are clean (containing photons or leptons) and has appreciable production rates, viz. $b \bar{b} \gamma \gamma$, $b \bar{b} \tau \tau$, fully leptonic and semi-leptonic $b \bar{b} W W^{*}$ and $W W^{*} \gamma \gamma$, and the $4 W$ channel with 2,3 and 4 lepton final states. We simulate the di-Higgs signal in these channels and the corresponding background processes in MG5_aMC@NLO [3]. The generated processes are showered and hadronised via Pythia [4]. These events are passed to Delphes-3.4.1 [5] for the detector simulation.

We perform a cut-based and multivariate analysis using Boosted Decision Tree (BDT) algorithm in the TMVA framework [6] in all the aforementioned di-Higgs channels. The $b \bar{b} \gamma \gamma$ channel contains photons in the final state. This channel has the disadvantage of $h \rightarrow \gamma \gamma$ branching ratio but has a clean signature at the collider. The QCD-QED $b \bar{b} \gamma \gamma$ process is the dominant background in this channel. We first do the cut based analysis where one of the kinematic variables is the invariant mass of the bottom pair, $m_{b b}$. The kinematic distribution of $m_{b b}$ is shown in Fig. 1 . The black curve corresponds to the di-Higgs signal, and others are the backgrounds. A cut of 100 $\mathrm{GeV}<m_{b b}<150 \mathrm{GeV}$ is applied to separate signal from backgrounds. We use 14 kinematic variables constructed from the final state during BDT analysis, and the final result improves about $20 \%$ compared to the previous cut-based analysis.

Next, we analyse the $b \bar{b} \tau \tau$ channel where we divide the final state according to the leptonic or hadronic decays of $\tau$ 's. The di- $\tau$ mass reconstruction is crucial in this channel because of the neutrinos in the final state. We use the collinear mass approximation technique to reconstruct the $h \rightarrow \tau \tau$ leg. The kinematic distribution of this variable, $M_{\tau_{h} \tau_{h}}$, is shown in Fig. 1. In case of $b \bar{b} W W^{*}$ and $W W^{*} \gamma \gamma$ final states, we divide them into fully-leptonic and semi-leptonic channels. The dominant background contribution comes from the $t \bar{t}$ and $t \bar{t} h, h \rightarrow \gamma \gamma$ process, respectively. In Fig. 1, the $m_{l l}$ and $\Delta R_{l j}$ corresponds to one of the best kinematic variables during the BDT analysis 
in $b \bar{b} W W^{*}$ and $W W^{*} \gamma \gamma$ channels, respectively. We further analyse the $W W^{*} W W^{*}$ channel in three final states, viz. same-sign di-lepton channel: $\ell^{ \pm} \ell^{ \pm}+4 j+\mathbb{E}_{T}, 3$-lepton channel: $3 \ell+2 j+\mathbb{E}_{T}$ and 4-lepton channel: $4 \ell+\mathbb{E}_{T}$. The final states with more leptons suffer from the event rates, while with more jets the QCD backgrounds become huge. In the same-sign di-lepton channel, we perform a BDT analysis and one best kinematic variable, $\Delta R_{l_{1} j_{2}}$, is shown in Fig. 1.
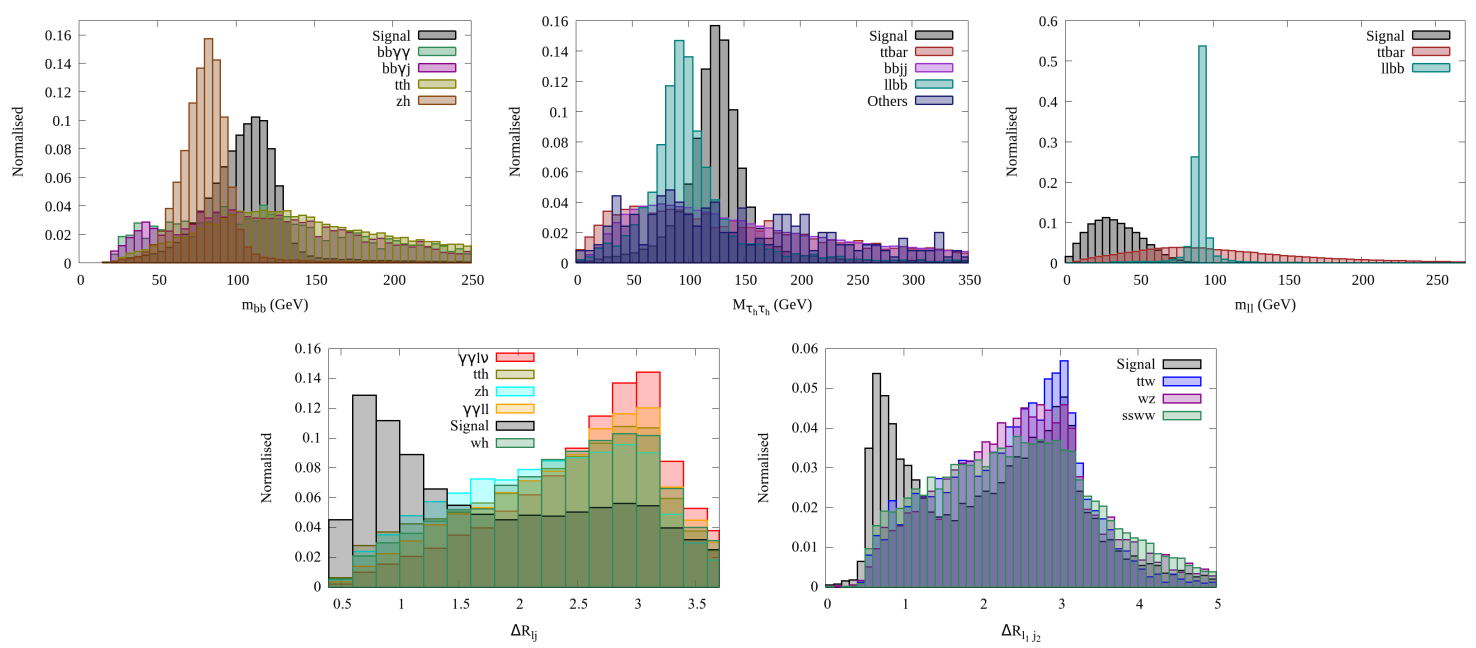

Figure 1: The normalised kinematic distributions for one of the best variables in various di-Higgs final states. The kinematic variables $m_{b b}, M_{\tau_{h} \tau_{h}}, m_{l l}, \Delta R_{l j}$ and $\Delta R_{l_{1} j_{2}}$ are taken from $b \bar{b} \gamma \gamma, b \bar{b} \tau \tau, b \bar{b} W W^{*}, W W^{*} \gamma \gamma$ and $4 W$ final states, respectively. The black curve corresponds to the signal process, $g g \rightarrow h h$. These distributions show the efficiency of these variables in distinguishing signal from the background processes.

Various non-SM or new physics (NP) might affect the di-Higgs production in many ways. One of them is via changing the self-coupling of Higgs boson. A deviation from the SM Higgs self-coupling value is quantified as the ratio of the measured value and the SM value of Higgs self-coupling, $\kappa=\lambda / \lambda_{S M}$. We analyse the best channel $b \bar{b} \gamma \gamma$ by selecting $\kappa=-1,1,2,5$ and 7 . Here, $\kappa=1$ corresponds to the SM Higgs self-coupling. The kinematics changes upon changing the $\kappa$ from SM value, which can be seen in Fig. 2 for $p_{T, \gamma \gamma}$ distribution. After using the BDT optimisation of $\kappa=1$ for all other values of $\kappa$, the log-likelihood confidence level (CL) hypothesis test [7] puts a limit of $-0.63<\kappa<8.07$ at $95 \%$ CL.

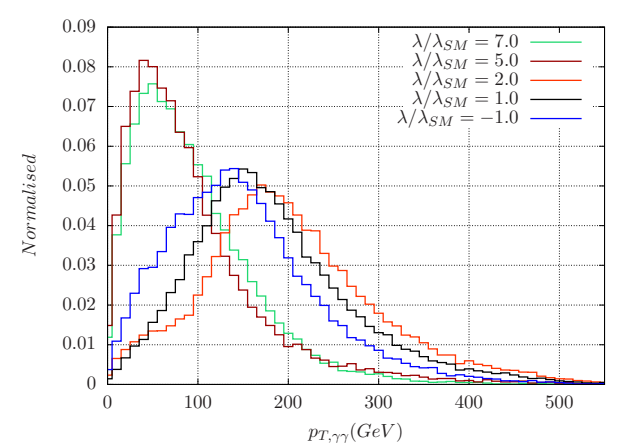

Figure 2: The normalised distribution of $p_{T, \gamma \gamma}$ in the $b \bar{b} \gamma \gamma$ channel for different values of $\lambda / \lambda_{S M}$. 


\section{The resonant Higgs pair production}

The Higgs sector in many BSM models includes additional Higgs bosons and the $125 \mathrm{GeV}$ Higgs boson. We consider a well-motivated BSM model, called MSSM, which contains two CP even $(h, H)$, one $\mathrm{CP}$ odd $(A)$ and two charged $\left(H^{ \pm}\right)$Higgs bosons. Here, the lightest $\mathrm{CP}$ even scalar, $\mathrm{h}$, is the SM-like Higgs boson. Our objective is to analyse the heavy Higgs $(H, A)$ decays, put upper limits (UL) on their production rates in a model-independent way and constrain the MSSM parameter space.

The following final states are considered in the analysis, viz. $p p \rightarrow H \rightarrow h h, p p \rightarrow H \rightarrow t \bar{t}$ and $p p \rightarrow b \bar{b} H, H \rightarrow \tau \tau$. Here, the first decay channel of heavy Higgs, $p p \rightarrow H \rightarrow h h$, is called resonant Higgs pair production. We select the following resonant di-Higgs decay modes, viz. $b \bar{b} \gamma \gamma, b \bar{b} b \bar{b}, b \bar{b} \tau \tau, b \bar{b} W W^{*}$ and $\gamma \gamma W W^{*}$. The main advantage is the resonant peak in the kinematic distribution, as compared to the previous non-resonant di-Higgs production (section 2). A comparison is shown for resonant process with various heavy Higgs masses and the non-resonant di-Higgs process at the leftmost plot of $p_{T, \gamma \gamma}$ in Fig. 3. In case of the $b \bar{b} \gamma \gamma$ channel, we do a cut-based analysis where we optimise over $m_{b \bar{b} \gamma \gamma}$ and $p_{T, \gamma \gamma}$ variables. Two plots on the right of Fig. 3 shows their kinematic distribution. The solid and dashed lines correspond to signal and backgrounds, respectively. We put model independent upper limits on the $\sigma(p p \rightarrow H \rightarrow h h)$, at 95\% CL. These limits are shown in Fig. 4 without (left) and with 5\% (right) systematic uncertainty. The limit is stronger below and above around $m_{H}=600 \mathrm{GeV}$ for the $H \rightarrow h h \rightarrow b \bar{b} \gamma \gamma$ and $H \rightarrow h h \rightarrow b \bar{b} b \bar{b}$ channel, respectively.
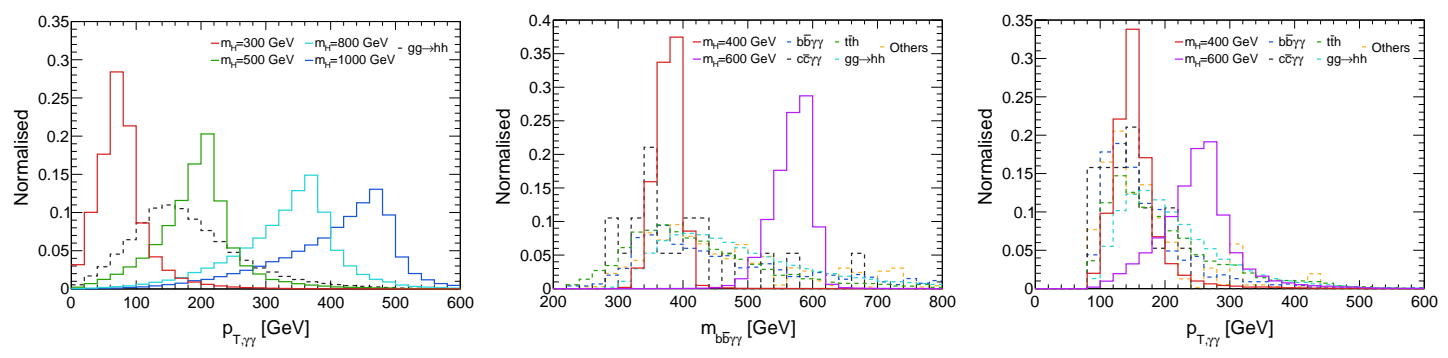

Figure 3: The leftmost distribution shows the difference in $p_{T, \gamma \gamma}$ between the resonant (with $m_{H}=$ $300,500,800,1000 \mathrm{GeV}$ ) and non-resonant Higgs pair production. The two distributions on the right corresponds to the best kinematic variables in the $p p \rightarrow H \rightarrow h h \rightarrow b \bar{b} \gamma \gamma$ final state, viz. $m_{b \bar{b} \gamma \gamma}$ and $p_{T, \gamma \gamma}$.

In case of $p p \rightarrow H \rightarrow t \bar{t}$ channel, the dominant background comes from $t \bar{t}$ process. Here, the final state for signal and background is similar $(t \bar{t})$ and this leads to difficulty in the signalbackground separation unless we reconstruct the resonant $H \rightarrow t \bar{t}$. This reconstruction is possible in case of the semi-leptonic channel where the only source of missing transverse energy, $\mathbb{E}_{T}$, is the leptonically decaying $W$ boson. We get two possible invariant masses, $m_{t \bar{t} 1}$ and $m_{t \bar{t} 2}$, for the two possible solutions of neutrino $p_{z}$. They are shown in Fig. 5. These variables perform better during the BDT analysis. The semi-leptonic $H \rightarrow t \bar{t}$ final state puts stronger UL on $\sigma(p p \rightarrow H \rightarrow t \bar{t})$ varying in the range $\sim[187,33]$ fb for $m_{H}=[400,1000] \mathrm{GeV}$. Finally, the $p p \rightarrow b \bar{b} H, H \rightarrow \tau \tau$ final state gives 95\% CL upper limit on $\sigma(p p \rightarrow b \bar{b} H) \times B r(H \rightarrow \tau \tau) \sim[22,4]$ fb between $m_{H}=[300,500] \mathrm{GeV}$. 

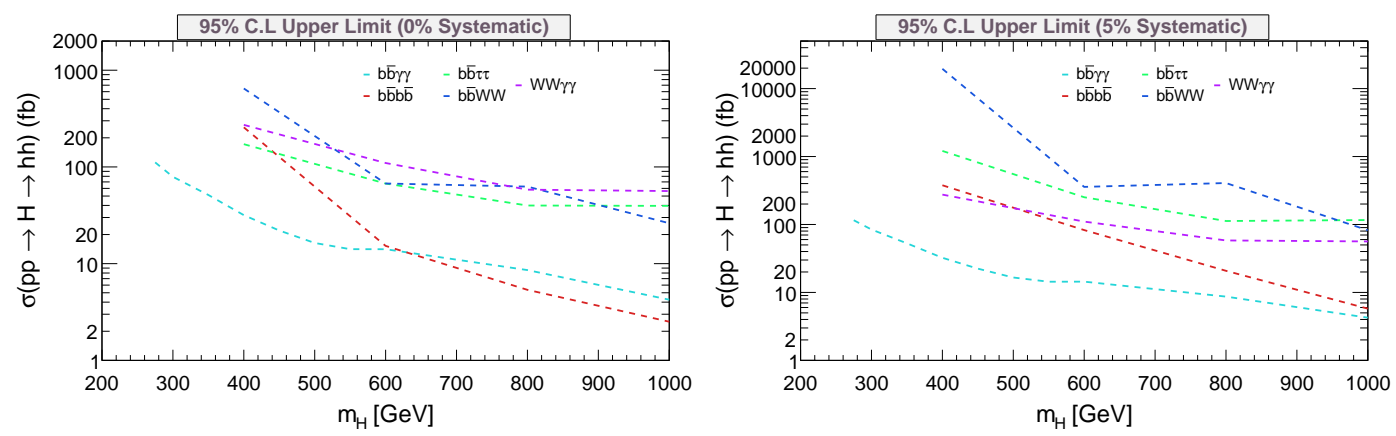

Figure 4: The 95\% CL upper limit placed on the resonant di-Higgs production cross-section for various final states without (left) and with 5\% (right) systematic uncertainty.
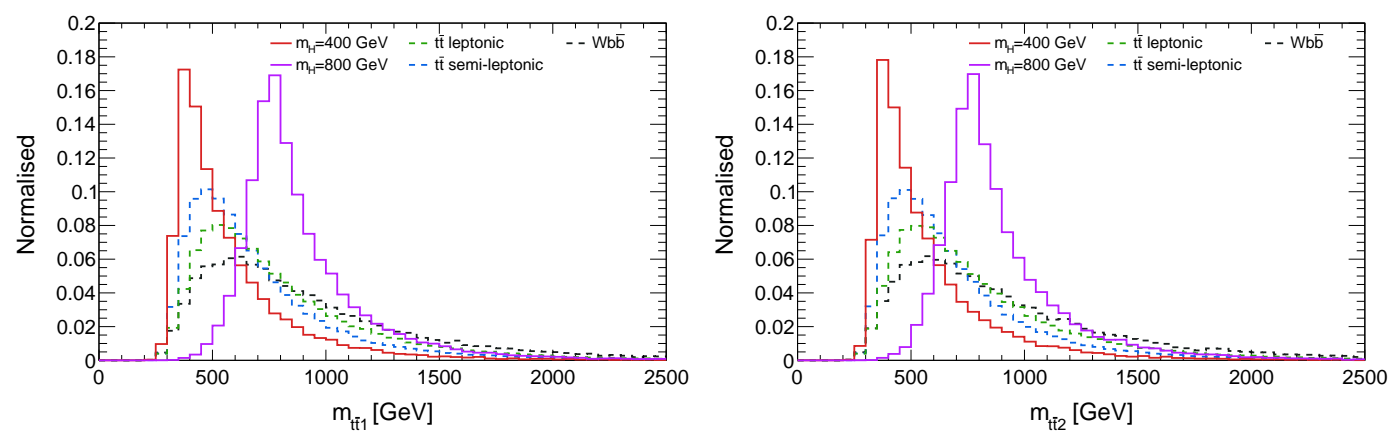

Figure 5: The invariant mass distribution reconstructed from the pair of top-quarks in the $H \rightarrow t \bar{t}$ (semileptonic) channel. The $m_{t \bar{t} 1}$ and $m_{t \bar{t} 2}$ correspond to the $t \bar{t}$ invariant mass from two possible solutions of neutrino transverse momentum in the semi-leptonic channel.
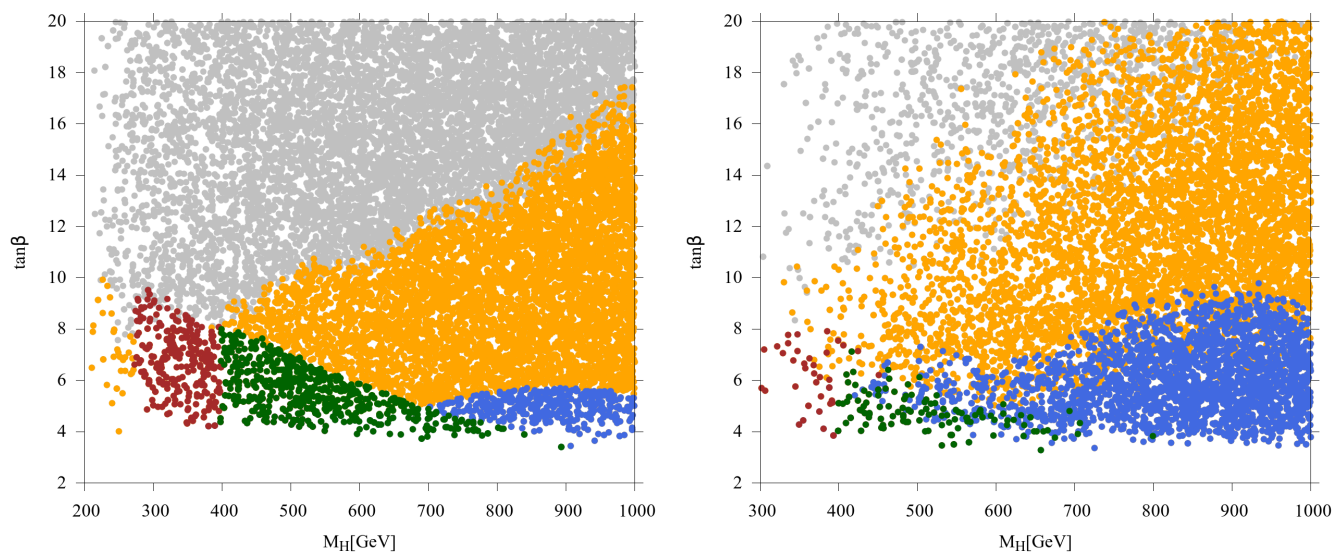

Figure 6: Projected HL-LHC reach for heavy Higgs decaying to only SM final states (left) and $\mathrm{SM}+$ supersymmetic decays (right) in the $m_{A}-\tan \beta$ plane.

Next, we translate the $95 \%$ CL upper limits into projected reach in the $m_{A}-\tan \beta$ plane, where $m_{A}$ is the pseudoscalar Higgs mass and $\tan \beta$ is the ratio of two vacuum expectation values of the two Higgs doublets in MSSM. In Fig. 6, the left plot shows the HL-LHC reach for the heavy Higgs searches. ATLAS and CMS Run-II data $\left(36 f^{-1}\right)$ excludes the grey coloured points via search in 
the $p p \rightarrow b \bar{b} H / A, H / A \rightarrow \tau \tau$ channel. The HL-LHC can constrain the brown, green and orange coloured points at $95 \% \mathrm{CL}$ via searches in $H \rightarrow h h \rightarrow b \bar{b} \gamma \gamma, H \rightarrow t \bar{t}$ and $b \bar{b} H, H \rightarrow \tau \tau$ final states, respectively. While the blue coloured points will evade these limits. These limits become weaker in the presence of non-SM decay modes of heavy Higgs boson, for example, heavy Higgs to neutralino/chargino decay, which is shown on the right in Fig. 6.

\section{Summary}

We have explored many di-Higgs final states, including better performing channels and the less-studied final states. The $W W^{*} \gamma \gamma$ channel has a good signal over background ratio, so that this channel might become important with higher energy colliders. Although a higher energy collider will be better in Higgs self-coupling measurement, we can get an improved result at the HL-LHC by combining several Higgs pair production final states and combining the results from both the ATLAS and CMS collaboration. We further performed heavy Higgs searches at the HL-LHC and put limits in the $m_{A}-\tan \beta$ plane. The $p p \rightarrow H \rightarrow h h$ and $p p \rightarrow H \rightarrow t \bar{t}$ final state can probe the low $m_{A}$ and low $\tan \beta$ region, whereas the high $\tan \beta$ region can be probed via the $p p \rightarrow b \bar{b} H, H \rightarrow \tau \tau$ channel. These limits get modified in the presence of non-SM heavy Higgs decays.

\section{References}

[1] A. Adhikary, S. Banerjee, R. K. Barman, B. Bhattacherjee and S. Niyogi, Revisiting the non-resonant Higgs pair production at the HL-LHC, JHEP 07 (2018) 116

[2] A. Adhikary, S. Banerjee, R. K. Barman and B. Bhattacherjee, Resonant heavy Higgs searches at the HL-LHC, JHEP 09 (2019) 068

[3] J. Alwall, R. Frederix, S. Frixione, V. Hirschi, F. Maltoni, O. Mattelaer et al., The automated computation of tree-level and next-to-leading order differential cross sections, and their matching to parton shower simulations, JHEP 07 (2014) 079

[4] T. Sjostrand, S. Ask, J. R. Christiansen, R. Corke, N. Desai, P. Ilten et al., An Introduction to PYTHIA 8.2, Comput. Phys. Commun. 191 (2015) 159-177

[5] DELPHES 3 collaboration, J. de Favereau, C. Delaere, P. Demin, A. Giammanco, V. Lema^ ${ }^{\wedge}$ tre, A. Mertens et al., DELPHES 3, A modular framework for fast simulation of a generic collider experiment, JHEP 02 (2014) 057

[6] A. Hoecker, P. Speckmayer, J. Stelzer, J. Therhaag, E. von Toerne, H. Voss et al., TMVA Toolkit for Multivariate Data Analysis, ArXiv Physics e-prints (Mar., 2007), [physics/0703039]

[7] T. Junk, Confidence level computation for combining searches with small statistics, Nucl. Instrum. Meth. A 434 (1999) 435, [hep-ex/9902006] 